\title{
Respiratory Modulation in Permanent Atrial Fibrillation
}

\author{
Mostafa Abdollahpur ${ }^{1}$, Fredrik Holmqvist ${ }^{2}$, Pyotr G.Platonov ${ }^{2}$, Frida Sandberg ${ }^{1}$ \\ ${ }^{1}$ Department of Biomedical Engineering, Lund University, Lund, Sweden \\ ${ }^{2}$ Skåne University Hospital, Lund, Sweden
}

\begin{abstract}
Several studies have shown that the autonomic nervous system (ANS) can induce changes during atrial fibrillation $(A F)$. There is currently a lack of methods for quantifying ANS induced variations during AF. The purpose of this study is to quantify respiratory induced modulation in the $f$-wave frequency trend. Following qrst-cancellation, the local $f$-wave frequency is estimated by fitting a harmonic $f$-wave model signal and a quality index (SQI) is computed based on the model fit. The resulting frequency trend is filtered using a narrow bandpass filter with a center frequency corresponding to the local respiration rate. The magnitude of the respiratory induced $f$-wave frequency modulation is estimated by the envelope of the filtered frequency trend. The performance of the method is validated using simulations and the method is applied to analyze $E C G$ data from eight patients with permanent $A F$ recorded during $0.125 \mathrm{~Hz}$ frequency controlled respiration before and after the full vagal blockade, respectively. Results from simulated data show the magnitude of the respiratory induced $f$-wave frequency modulation can be estimated with an error of less than $\epsilon=0.005 \mathrm{~Hz}$ if the SQI is above 0.45 . The signal quality was sufficient for analysis in 7 out of 8 patients. In 4 patients the magnitude decreased and in 3 patients there was no change.
\end{abstract}

\section{Introduction}

The autonomic nervous system (ANS) plays an important role in modulating cardiac arrhythmias [1]. Autonomic dysfunction is defined as one of the major health modifiers linked to atrial fibrillation (AF) onset[2]. During normal heart rhythm, heart rate variability can be used to analyse ANS function, but there is currently a lack of tools for analysis of ANS function during AF. Previous research has established that vagal simulation and sympathetic simulation can change the atrial action potential as well as the atrial refractory period[3]. The f-wave frequency is a fundamental characteristic of the atrial electrical activity during AF that can be estimated from the surface ECG and is inversely proportional to the atrial cycle length. Previ-

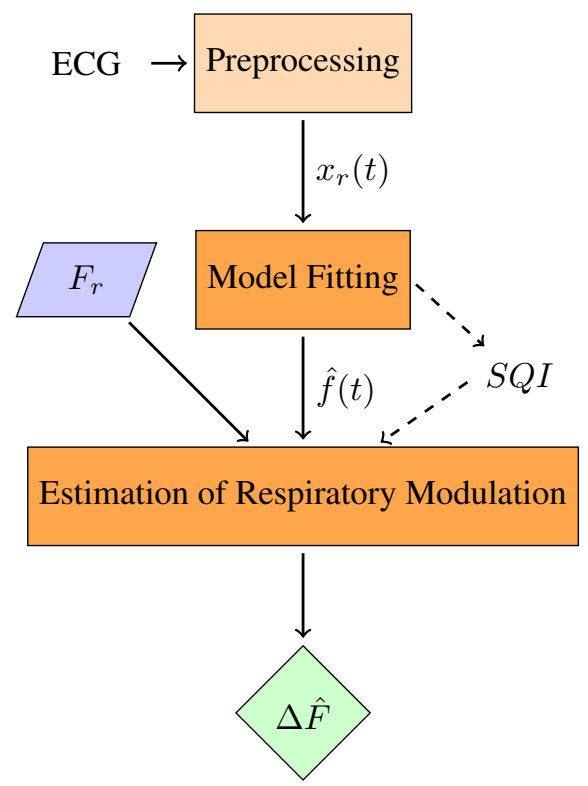

Figure 1. Overall structure of the proposed method

ous studies has shown that the f-wave frequency changes in response to head-up and head-down tilt-test [4], and that paced breathing can induce modulation in the f-wave frequency [5]. In this study, we propose a novel approach to quantify respiratory induced modulation in the f-wave frequency trend. This is challenging, since respiratory induced variations in the f-wave freqeuncy are small and largely concealed by other variations.

\section{Methods}

A schematic outline of the method is presented in Fig. 1. After extracting f-waves $x_{r}(t)$ from ECG by applying spatiotemporal QRST cancellation [6], a model-based approach is employed to estimate the local f-wave frequency [7]. If the f-wave signal quality is sufficient for analysis, respiratory modulation is obtained from the fwave frequency trend using bandpass filtering and envelope estimation. 


\subsection{Local f-wave Frequency}

The local f-wave frequency is estimated using a modelbased approach [7]. In this method, the Hilbert transform is employed to construct a complex-valued analytical signal $x_{c}(t)$ from the real-valued $x_{r}(t)$, and a model signal $s(t)$ is fitted to $x_{c}(t)$. The model signal is defined as the sum of a complex exponential signals with frequency $F_{0}$ and its harmonic,

$$
s\left(t ; F_{0}, A_{m}, \phi_{m}\right)=\sum_{m=1}^{2} A_{m} e^{j\left(m 2 \pi F_{0} t+\phi_{m}\right)},
$$

where $A_{m}$ and $\phi_{m}$ denotes the amplitude and phase, of the $m$ :th harmonic, respectively. The model is fitted to 0.5 second segments of $x_{c}(t)$ using maximum likelihood estimation to obtain $\hat{s}(t)$. The corresponding local frequency estmates $\hat{F}_{0}$ provides an estimate of the f-wave frequency trend $\hat{f}(t)$. The normalized RMS of the model error $x_{c}(t)-\hat{s}(t)$, is used to compute a signal quality index (SQI) [7]. The SQI can take values between 0 and 1, where 1 indicates a perfect model fit.

\subsection{Estimation of Respiratory Modulation}

A fourth-order Butterworth fixed bandwidth bandpass filter [8] with center frequency corresponding to the respiration rate $F_{r}$ and a bandwidth of $0.06 \mathrm{~Hz}$ is employed to extract respiratory modulation in the estimated AF frequency trend $\hat{f}(t)$. The envelope of the filtered signal is computed as the magnitude of its analytic signal obtained using Hilbert transformation. To obtain a robust estimate of the magnitude of the modulation the 5-min median is employed; this estimate is denoted $\Delta \hat{F}$.

\section{Datasets}

Simulated data is used to validate the method, and determine the SQI required for accurate estimation of the magnitude of the f-wave frequency modulation. The method is applied to analyze changes in respiratory induced f-wave frequency modulation in clinical data from a study involving AF patients during controlled breathing before and after full vagal blockade. [5].

\subsection{Simulated Data}

A modified version of the saw-tooth f-wave model [9] is employed for the simulations. The simulated f-waves consists of a sinusoid and its first harmonic,

$$
x(t)=\sum_{k=1}^{2} a_{k} \sin \left(2 \pi k f_{0} t+k \frac{\Delta F}{F_{r}} \sin \left(2 \pi F_{r} t\right)+\Phi(t)\right)+v(t),
$$

where $k=1,2$ denotes number of the harmonic, $a_{k}$ specifies the amplitude of the $k$ :th harmonic, and $f_{0}, F_{r}$, and $\triangle F$ define the fundamental $\mathrm{f}$-wave frequency, the respiration frequency, and the maximum frequency deviation in response to respiration, respectively. Random phase variation $\Phi(t)$ corresponding to white Gaussian noise with standard deviation $\sigma_{\Phi}$ is added to model other variations in the f-wave frequency. White Gaussian noise $v(t)$ with standard deviation $0.1 * a_{1}$ is added to form realistic f-wave signals. The simulated signal $x(t)$ is sampled at the rate of $50 \mathrm{~Hz}$. The following parameters are used for the simulations: $f_{0}=7 \mathrm{~Hz}, F_{r}=0.125 \mathrm{~Hz}, \triangle F=0.15 \mathrm{~Hz}$, and $a_{k}$ chosen such that the standard deviation of $x(t), \sigma_{x}=50$ $\mu \mathrm{V}$. The magnitude of the random frequency variation is changed by setting $\sigma_{\Phi}$ to values between 0 and 0.9 . Examples of simulated f-waves with different $\sigma_{\Phi}$ are displayed in Fig. 2.

(a)

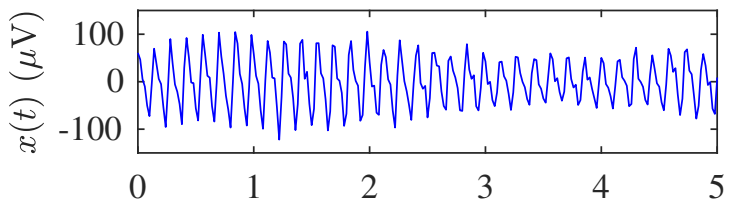

(b)

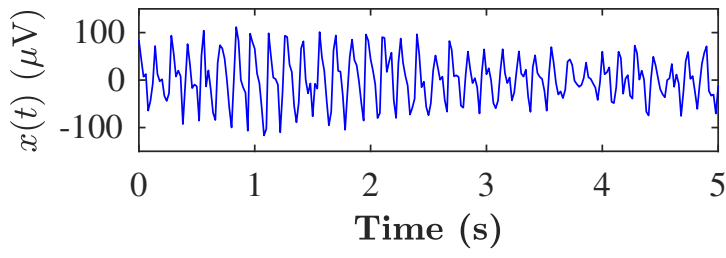

Figure 2. Example of simulated f-wave signals with (a) $\sigma_{\Phi}=0$ and (b) $\sigma_{\Phi}=0.45$.

\subsection{Clinical Data}

The method was applied to analyse data from a clinical study involving eight patients with permanent AF and pacemaker; the details of the study protocol is described in [5]. The patients were asked to breath at a fixed rate of $0.125 \mathrm{~Hz}$, and ECG signals were recorded during a 5minute period when the respiratory pattern was stable. Full vagal blockade was induced by intravenous administration of atropine, the patients were again asked to breath at a fixed rate of $0.125 \mathrm{~Hz}$ and ECG signals were recorded for another 5-minute period. The recordings are referred to as Controlled Respiration (CR) and Post Atropine (PA), respectively. 


\section{Results}

\subsection{Simulated Signals}

The proposed method was applied to estimate the magnitude of the respiratory modulation from 5 minuets segments of simulated data. Results shows that increasing the random frequency variation by increasing $\sigma_{\Phi}$ gives a decrease of the SQI, c.f. Fig 3. Further, the estimation error between true and estimated magnitude of the respiratory modulation, quantified by the absolute deviation $|\triangle F-\triangle \hat{F}|$, is displayed in Fig. 4 as function of the SQI. This result suggest that the magnitude of the respiratory induced f-wave frequency modulation can be estimated with an error of less than $\epsilon=0.005 \mathrm{~Hz}$ if the SQI is below $\Gamma_{S Q I}=0.45$. For SQI $>\Gamma_{S Q I}$ the estimation error becomes large which indicates that the estimate $\triangle \hat{F}$ is unreliable.

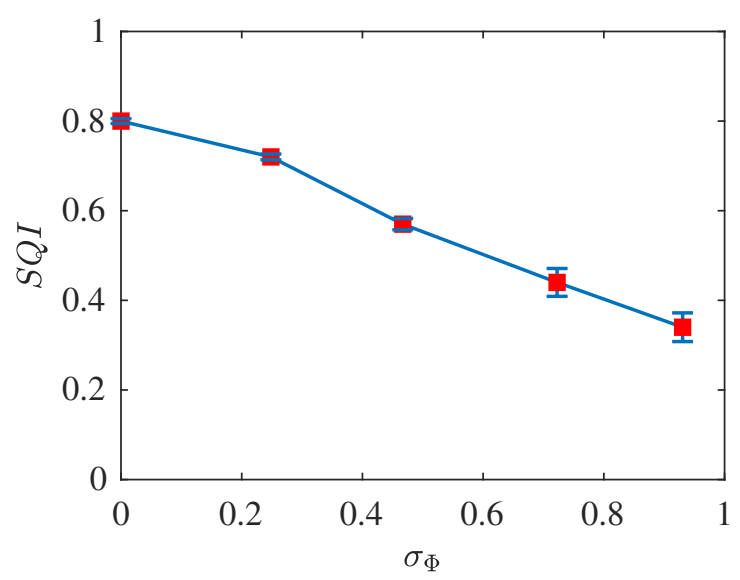

Figure 3. Resulting SQI when the local f-wave frequency is estimated from simulated data with different values of $\sigma_{\Phi}$

\subsection{Clinical Data}

The signal quality was sufficient for analysis, i.e., $S Q I>\Gamma_{S Q I}$, in 7 out of 8 patients. The estimated f-wave frequency trend and its corresponding respiratory modulation for one of these patient during CR and PA, respectively, is displayed in Fig. 5. For this patient, $\Delta \hat{F}$ decreased from $0.167 \mathrm{~Hz}$ to 0.126 in response to the vagal blockade.

The magnitude of the respiratory frequency modulation was $0.16 \pm 0.01 \mathrm{~Hz}$ (mean \pm std) during CR $0.14 \pm 0.02$ $\mathrm{Hz}$ during PA. In four patients $\Delta \hat{F}$ decreased in response to atropine (difference $>\epsilon$ ) and in three patients there was no change (difference $<\epsilon$ ). The results from analysis of the clinical data are summarized in Table 1.

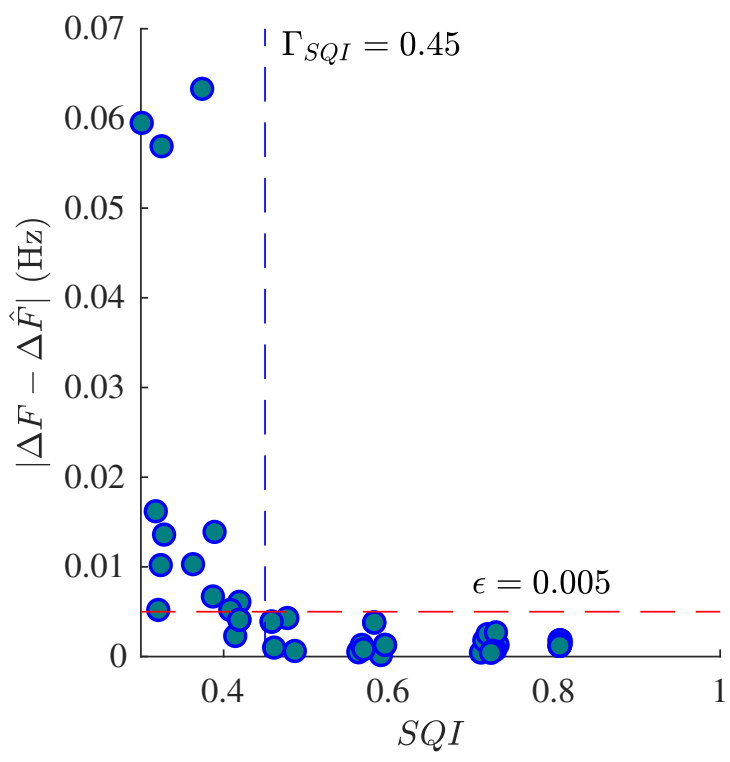

Figure 4. Estimation error in simulated data, quantified by $|\Delta F-\Delta \hat{F}|$ plotted as function of the corresponding SQI.

\section{Discussion and Conclusion}

The proposed method can be used to quantify respiratory modulation in the f-wave frequency trend. Our results from clinical data suggest that such modulation can be partly attributed to parasympathetic regulation in the atria during AF.

Sufficient signal quality is crucial for the analysis; our simulation results shows that respiratory modulation cannot be accurately estimated when the SQI above 0.45. Accurate estimation of respiratory modulation in the f-wave frequency trend requires a larger SQI than the threshold previously proposed in [7] when the aim was to improve AF detection.

In this study, the respiration rate is assumed to be known and fixed. However, the methodology can be extended to incorporate ECG derived estimation of the respiration rate and the bandpass filter can be adapted to account for timevarying respiration rates.

\section{Acknowledgments}

This project was funded by the European Union's Horizon 2020 research and innovation programme under the Marie Skłodowska-Curie grant agreement No.766082.

\section{References}

[1] Shen MJ, Zipes DP. Role of the autonomic nervous system in modulating cardiac arrhythmias. Circulation research 2014; 114(6):1004-1021.

[2] Fabritz L, Guasch E, Antoniades C, Bardinet I, Benninger 
$(a)$

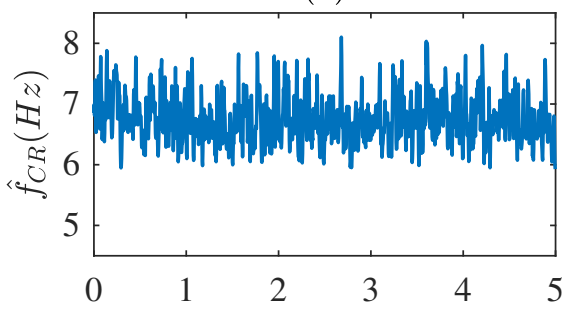

(c)

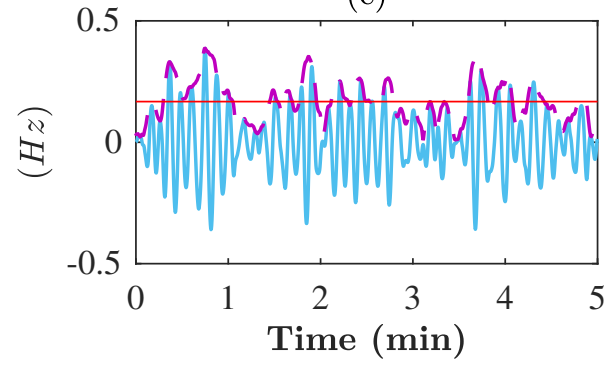

(b)

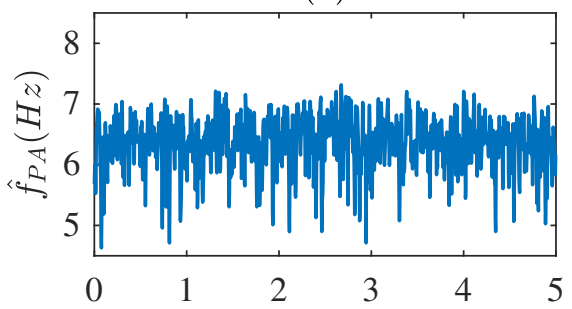

(d)

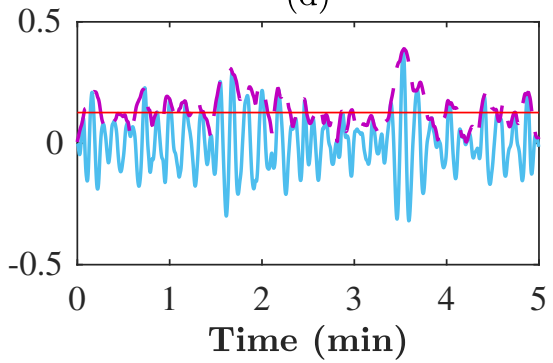

Figure 5. Results from analysis of ECG data from patient d in (a,c) CR and (b,d) PA, respectively. (a-b) Estimated $\hat{f}(t)$ and (c-d) corresponding (solid blue) filtered $\hat{f}(t)$, (dashed purple) its envelope and (solid red) estimated $\Delta \hat{F}$. For this patient, $\Delta \hat{F}_{C R}=0.167 \mathrm{~Hz}$, and $\Delta \hat{F}_{P A}=0.126 \mathrm{~Hz}$.

Table 1. Results from clinical data (CR: Controlled Respiration, PA: Post Atropine). Arrows indicate difference $>\epsilon$. Dotts indicate SQI insufficient for analysis.

\begin{tabular}{cccc}
\hline \hline Patient & Phase & SQI & $\triangle \hat{F}$ \\
\hline \multirow{2}{*}{$\mathrm{a}$} & $\mathrm{CR}$ & 0.64 & 0.147 \\
& PA & 0.54 & $0.138^{\downarrow}$ \\
\hline \multirow{2}{*}{$\mathrm{b}$} & $\mathrm{CR}$ & 0.41 & $\ldots$ \\
& $\mathrm{PA}$ & 0.40 & $\ldots$ \\
\hline \multirow{2}{*}{$\mathrm{c}$} & $\mathrm{CR}$ & 0.62 & 0.143 \\
& PA & 0.57 & 0.117 \\
\hline \multirow{2}{*}{$\mathrm{d}$} & $\mathrm{CR}$ & 0.59 & 0.167 \\
& PA & 0.57 & 0.126 \\
\hline \multirow{2}{*}{$\mathrm{e}$} & $\mathrm{CR}$ & 0.55 & 0.166 \\
& $\mathrm{PA}$ & 0.53 & 0.165 \\
\hline \multirow{2}{*}{$\mathrm{f}$} & $\mathrm{CR}$ & 0.54 & 0.165 \\
& $\mathrm{PA}$ & 0.53 & 0.165 \\
\hline \multirow{2}{*}{$\mathrm{g}$} & $\mathrm{CR}$ & 0.55 & 0.157 \\
& $\mathrm{PA}$ & 0.53 & 0.158 \\
\hline \multirow{2}{*}{$\mathrm{h}$} & $\mathrm{CR}$ & 0.56 & $0.156 \downarrow$ \\
& PA & 0.57 & $0.142^{\downarrow}$ \\
\hline \hline
\end{tabular}

G, Betts TR, Brand E, Breithardt G, Bucklar-Suchankova G, Camm AJ, et al. Defining the major health modifiers causing atrial fibrillation: a roadmap to underpin personalized prevention and treatment. Nature Reviews Cardiology 2016; 13(4):230-237.

[3] Chen PS, Chen LS, Fishbein MC, Lin SF, Nattel S. Role of the autonomic nervous system in atrial fibrillation: pathophysiology and therapy. Circulation research 2014; 114(9):1500-1515.

[4] Östenson S, Corino VD, Carlsson J, Platonov PG. Autonomic influence on atrial fibrillatory process: head-up and head-down tilting. Annals of Noninvasive Electrocardiology 2017;22(2):e12405.

[5] Holmqvist F, Stridh M, Waktare JE, Brandt J, Sornmo L, Roijer A, Meurling CJ. Rapid fluctuations in atrial fibrillatory electrophysiology detected during controlled respiration. American Journal of Physiology Heart and Circulatory Physiology 2005;289(2):H754-H760.

[6] Stridh M, Sornmo L. Spatiotemporal qrst cancellation techniques for analysis of atrial fibrillation. IEEE Transactions on Biomedical Engineering 2001;48(1):105-111.

[7] Henriksson M, Petrènas A, Marozas V, Sandberg F, Sörnmo L. Model-based assessment of f-wave signal quality in patients with atrial fibrillation. IEEE Transactions on Biomedical Engineering 2018;65(11):2600-2611.

[8] Kumar RR, Pal RN. A gradient algorithm for centerfrequency adaptive recursive bandpass filters. Proceedings of the IEEE 1985;73(2):371-372.

[9] Petrenas A, Marozas V, Sornmo L, Lukosevicius A. An echo state neural network for qrst cancellation during atrial fibrillation. IEEE transactions on biomedical engineering 2012; 59(10):2950-2957.

Address for correspondence:

Mostafa Abdollahpur

Department of Biomedical Engineering, Lund University

SE-22100 Lund, sweden

mostafa.abdollahpur@bme.lth.se 